Response of Germination and Seedling Development of Cotton to Salinity under Optimal and Suboptimal Temperatures

\author{
Nurgül ERGIN ${ }^{1}$, Engin Gökhan KULAN ${ }^{2}$, Mehmet Akif GÖKÜKARA ${ }^{3}$, Muhammed Fatih KAYA4 \\ Şaban Özgür ÇETIN ${ }^{5}$, Mehmet Demir KAYA ${ }^{6}$ \\ ${ }^{1}$ Department of Field Crops, Faculty of Agriculture and Natural Sciences, Bilecik Şeyh Edebali University, Bilecik, Turkey, 2,3,4,5,6Department \\ of Field Crops, Faculty of Agriculture, Eskişehir Osmangazi University, Eskişehir, Turkey \\ ${ }^{1}$ https://orcid.org/0000-0003-3105-7504, ${ }^{2}$ https://orcid.org/0000-0002-7147-6896, ${ }^{3}$ https://orcid.org/0000-0002-2019-1487 \\ ${ }^{4}$ https://orcid.org/0000-0002-0875-7302, ${ }^{5}$ https://orcid.org/0000-0002-1696-3819, ${ }^{6}$ https://orcid.org/0000-0002-4681-2464 \\ $\bowtie$ : nurgulergin180@gmail.com
}

\section{ABSTRACT}

The objective of the study was to investigate the effects of salinity stresses $(50,100,150,200$ and $250 \mathrm{mM}$ of $\mathrm{NaCl})$ on germination and early seedling growth of six cotton varieties (Lydia, Carisma, Flash, BA151, BA525 and ST468) under optimum $\left(25^{\circ} \mathrm{C}\right)$ and low temperature $\left(18^{\circ} \mathrm{C}\right)$ conditions. Germination percentage, mean germination time, germination index, germination stress tolerance index, seedling length, vigor index, seedling fresh and dry weight of cotton varieties were investigated. Results showed that low temperature led to decreasing in germination and seedling growth, and caused retardation of mean germination time. Under suboptimal temperature, germination percentage reduced from $86.3 \%$ to $77.8 \%$ and seedling length decreased from $12.02 \mathrm{~cm}$ to $5.36 \mathrm{~cm}$. Each increase in salinity levels higher than $50 \mathrm{mM}$ resulted in decreasing in germination and seedling growth parameters of cotton varieties. No seedling growth was observed at $250 \mathrm{mM}$ of $\mathrm{NaCl}$ at $18^{\circ} \mathrm{C}$. Cotton varieties showed different tolerance levels to salinity, while they could tolerate it up to $100 \mathrm{mM}$. It was concluded that Flash and ST468 varieties exhibited better performance under salinity stresses both at optimal and suboptimal temperatures.

\section{Research Article}

$\begin{array}{ll}\text { Article History } & \\ \text { Received } & : 28.04 .2020 \\ \text { Accepted } & : 04.07 .2020\end{array}$

\author{
Keywords \\ Cold \\ Cultivar \\ Germination \\ Gossypium hirsutum L. \\ $\mathrm{NaCl}$
}

\title{
Normal ve Düşük Sıcaklıklarda Pamuğun Çimlenme ve Fide Gelişimine Tuzluluğun Etkisi
}

\section{ÖZET}

$\mathrm{Bu}$ araştırma, farkl tuz dozlarının (50, 100, 150, 200 ve $250 \mathrm{mM}$ $\mathrm{NaCl}$ ) altı pamuk çeşidinin (Lydia, Carisma, Flash, BA151, BA525 ve ST468) optimum $\left(25^{\circ} \mathrm{C}\right)$ ve düşük sıcaklıkta $\left(18^{\circ} \mathrm{C}\right)$, çimlenme ve erken fide büyümesine etkilerini belirlemek amaciyla yürütülmüştür. Araştırmada pamuk çeşitlerinin çimlenme yüzdesi, ortalama çimlenme süresi, çimlenme indeksi, çimlenme stres tolerans indeksi, fide uzunluğu, fide yaş ve kuru ağırlıkları belirlenmiştir. Sonuçlar, düşük sıcaklığın çimlenme ve fide büyümesinde azalmalara yol açtığını ve ortalama çimlenme süresinin gecikmesine neden olduğunu göstermiştir. Düşük sıcaklıkta, çimlenme yüzdesinin $\% 86.3$ 'ten $\% 77.8$ 'e ve fide uzunluğunun $12.02 \mathrm{~cm}$ 'den $5.36 \mathrm{~cm}$ 'ye düştüğü belirlenmiştir. Tuz dozlarındaki $50 \mathrm{mM}$ 'den yüksek her artış, pamuk çeşitlerinin çimlenme ve fide gelişiminde azalmalara neden olmuştur. Ayrıca $18^{\circ}$ C'de $250 \mathrm{mM}$ tuz dozunda fide gelişimi gözlenmemiştir. Pamuk çeşitleri, tuzluluğa farklı tolerans seviyeleri gösterirken, pamuğun 100 mM'ye kadar tuzluluğu tolere edebildiği belirlenmiştir. Flash ve ST468 çeşitlerinin hem optimum hem de düşük sıcaklıklarda tuzluluk stresleri altında daha iyi performans gösterdiği sonucuna varılmıştır.

\section{Araştırma Makalesi}

\section{Makale Tarihçesi}

Geliş Tarihi : 28.04 .2020

Kabul Tarihi : 04.07 .2020

\section{Anahtar Kelimeler}

Soğuk

Çeşit

Çimlenme

Gossypium hirsutum L.

$\mathrm{NaCl}$.

\footnotetext{
To Cite : Ergin N, Kulan EG, Gözükara MA, Kaya MF, Çetin ŞÖ, Kaya MD 2021. Response of Germination and Seedling Development of Cotton to Salinity under Optimal and Suboptimal Temperatures. KSU J. Agric Nat 24 (1): 108115. https://doi.org/10.18016/ksutarimdoga.vi.728814.
} 


\section{INTRODUCTION}

Cotton (Gossypium hirsutum L.) is the most important fiber crop and is extensively cultivated for textile industry in the Aegean, Mediterranean and Southern Anatolian Regions of Turkey (Anonymous, 2020). Cotton is mainly produced for fiber, raw material of the ginning and textile industries, even oil and oil-cake are obtained from cottonseed. The oil of cottonseed is used for both edible oil and the production of biodiesel. Approximately $80 \%$ of world cotton production has been met by a few countries, including Turkey because of tropic or subtropic climatic requirements (Mert, 2017).

Soil salinity and low temperature are the most important stress factors that limit germination, seedling growth and the yield of cotton in Turkey and all over the world (Kalefetoğlu and Ekmekçi, 2005). Salinity risk in the areas of cotton cultivation has been continuously increased by intensive irrigation frequency and excessive water application, poor water management and high evaporation due to hot and dry weather during summer seasons. The salts in the soil prevent the water from entering the seed by either constituting osmotic pressure which creates a barrier to the seed or toxic effects caused by $\mathrm{Na}^{+}$and $\mathrm{Cl}^{-}$ions (Khajeh-Hosseini et al., 2003). Cotton is classified as a relatively salt tolerant plant by Fowler (1986), while there are differences between cotton varieties (Gosset et al., 1994). Vulkan-Levy et al. (1998) found that increasing salinity resulted in decreasing the seed cotton yield. Maas (1986) reported that cotton yield decreased around $50 \%$ at the level of $17 \mathrm{dS} \mathrm{m}^{-1}$. However, cotton plant is sensitive to low temperature during germination and low soil temperature delays germination and emergence, increases the risk of seedling diseases, and causes weak seedling development (Çokkizgin and Bölek, 2015). It was determined that root development and seedling growth of cotton were inhibited when the soil temperature drops below $14.5^{\circ} \mathrm{C}$ by Jackson (2012), but still farmers prefer earlier sowing to obtain higher yield and to avoid the lack of rainfall after planting. For these reasons, this research was undertaken to determine if there were genetic variations among cotton varieties under salinity stresses at low temperature in terms of germination and seedling growth.

\section{MATERIAL and METHODS}

A laboratory experiment was conducted at Department of Field Crops, Eskişehir Osmangazi University, Turkey. The seeds of six cotton varieties Lydia, Carisma, Flash, BA151, BA525 and ST468 were exposed to five concentrations of $\mathrm{NaCl}$ with 50 , $100,150,200$ and $250 \mathrm{mM}$ under optimal $\left(25^{\circ} \mathrm{C}\right)$ and suboptimal $\left(18^{\circ} \mathrm{C}\right)$ temperatures. Distilled water was used as a control treatment.

Germination test was performed by the procedures of ISTA (2003) rules with two hundred $(4 \times 50)$ seeds of each cotton variety for each treatment. Fifty seeds were inserted into three-layer filter papers irrigated with $7 \mathrm{~mL}$ of the respective solutions for each paper. After filter papers with seeds were rolled, they were placed into a sealed plastic bag to prevent moisture loss. Each rolled paper was renewed every 2 days after incubation to avoid the salt accumulation. The packages were incubated at optimal $25^{\circ} \mathrm{C}$ and suboptimal $18{ }^{\circ} \mathrm{C}$ conditions in the dark and a seed with $2 \mathrm{~mm}$ radicle was counted every $24 \mathrm{~h}$ for $12 \mathrm{~d}$ as germinated. Mean germination time (MGT) was calculated to evaluate the speed of germination as defined by ISTA (2003) rules. MGT $=\Sigma(\mathrm{Dn}) / \Sigma \mathrm{n}$, where, $\mathrm{n}$ is the seed number germinated on day $\mathrm{D}$, and $\mathrm{D}$ is the number of days from the beginning of germination test. Germination index (GI) was calculated according to the following formula. GI= Number of germinated seeds / days of first count +. . .+ Number of germinated seeds / days of final count (Salehzade et al., 2009). Germination stress tolerance index (GSTI, $\%)=\left[\mathrm{nd}_{2}(1.00)+\mathrm{nd}_{4}(0.75)+\mathrm{nd}_{6}(0.5)+\mathrm{nd}_{8}(0.25)\right.$ of stressed seeds / $\mathrm{nd}_{2}(1.00)+\mathrm{nd}_{4}(0.75)+\mathrm{nd}_{6}(0.5)+$ $\mathrm{nd} 8(0.25)$ of control seeds] $\times 100$, where $\mathrm{n}$ is the number of seeds germinated at day d (Ahmad et al., 2009). At $12^{\text {th }}$ day, ten seedlings selected randomly from each treatment were sampled for determination of seedling growth traits such as seedling length (SL), seedling fresh weight (SFW) and seedling dry weight (SDW). After the seedling fresh weight was directly weighed, the seedlings were transferred into an oven at $70^{\circ} \mathrm{C}$ for 48 hours for determination of dry weight.

The experimental data was statistically analyzed by two-factor factorial (salinity $\times$ variety) arranged in a completely randomized design (CRD) with 4 replicates for each temperature. Analysis of variance and comparison of means were performed by MSTATC program (Michigan State University v. 2.10).

\section{RESULTS and DISCUSSION}

The main effects of temperature, salinity and cotton variety were displayed in Table 1 . As expected, low temperature caused a significant decrease in germination percentage, germination index, germination stress tolerance index and seedling growth characteristics, while mean germination time delayed. Germination and seedling growth of cotton were restricted, but mean time to germination retarded when salinity concentration increased. The results confirmed the findings of Varghese et al. (1995) and Qadir and Shams (1997), who reported that germination and emergence of cotton varieties were reduced and delayed by increasing salinity. 
Table 1. Main effects of the temperature, salinity and variety on germination and seedling growth parameters of cotton $( \pm$ SEM)

Tablo 1. Pamuğun çimlenme ve fide büyüme parametreleri üzerine sıcaklık, tuzluluk ve çeşit etkileri cotton $( \pm$ Standart hata)

\begin{tabular}{|c|c|c|c|c|c|c|c|c|}
\hline Factors & GP & MGT & GI & GSTI & SL & SFW & SDW & DM \\
\hline \multicolumn{9}{|c|}{ Temperature } \\
\hline $18^{\circ} \mathrm{C}$ & $77.8 \pm 1.51^{b}$ & $83 \pm 0.71^{a}$ & $8.39 \pm 0,34 b$ & $56.0 \pm 3.11^{b}$ & $5.36 \pm 0.30^{b}$ & $190 \pm 9.56^{b}$ & $40.9 \pm 1.60^{b}$ & $62.8 \pm 2.44^{b^{*}}$ \\
\hline $25^{\circ} \mathrm{C}$ & $86.3 \pm 0.80 \mathrm{a}$ & $.19 \pm 0.08^{b}$ & $15.73 \pm 0,500^{a}$ & $75.4 \pm 1.79$ a & $12.02 \pm 0.39$ a & $381 \pm 11.10^{\mathrm{a}}$ & $48.8 \pm 0.41^{\mathrm{a}}$ & $85.4 \pm 0.48^{\mathrm{a}}$ \\
\hline \multicolumn{9}{|l|}{ Salinity } \\
\hline Control & $5^{a}$ & $08 \pm 0.13^{f}$ & $17.14 \pm 0.56^{a}$ & $100.0 \pm 14.43^{\mathrm{a}}$ & $12.46 \pm 0.69^{\mathrm{a}}$ & $439 \pm 17.75^{a}$ & $48.9 \pm 0.79$ a & $87.6 \pm 0.68$ a \\
\hline $50 \mathrm{mM}$ & & $3.35 \pm 0.16^{\mathrm{e}}$ & $15.60 \pm$ & $93.8 \pm 1.7$ & $12.81 \pm 0.83^{\mathrm{a}}$ & 413 & $0^{\text {a }}$ & 86.9 \\
\hline $100 \mathrm{mM}$ & $86.0 \pm 1.93 b$ & $3.71 \pm 0.15^{\mathrm{d}}$ & $13.40 \pm 0.61^{\mathrm{c}}$ & $78.0 \pm 1.88^{c}$ & $9.98 \pm 0.56^{b}$ & $321 \pm 15.53^{c}$ & $49.1 \pm 0.67$ a & $82.9 \pm 0.87^{b}$ \\
\hline $150 \mathrm{mM}$ & $84.5 \pm 1.21 \mathrm{~b}$ & $4.37 \pm 0.22^{\mathrm{c}}$ & $11.67 \pm 0.68^{d}$ & $59.5 \pm 2.58^{\mathrm{d}}$ & $7.89 \pm 0.46^{\mathrm{c}}$ & $236 \pm 12.00^{d}$ & $48.8 \pm 0.86^{a}$ & $76.8 \pm 1.18^{\mathrm{c}}$ \\
\hline $200 \mathrm{mM}$ & $80.3 \pm 1.66^{c}$ & $5.60 \pm 0.28 b$ & $8.58 \pm 0.54$ e & $39.8 \pm 2.97$ е & $5.74 \pm 0.40^{\mathrm{d}}$ & $197 \pm 10.88^{\mathrm{e}}$ & $48.7 \pm 1.03 \mathrm{a}$ & $71.8 \pm 1.44^{d}$ \\
\hline $250 \mathrm{mM}$ & $64.3 \pm 3.12^{\mathrm{d}}$ & $6.93 \pm 0.34^{\mathrm{a}}$ & $5.96 \pm 0.52^{f}$ & $23.1 \pm 3.00^{\mathrm{f}}$ & $3.19 \pm 0.47^{\mathrm{e}}$ & $106 \pm 15.59^{\mathrm{f}}$ & $24.1 \pm 3.52^{\mathrm{b}}$ & $38.5 \pm 5.57^{\mathrm{e}}$ \\
\hline \multicolumn{9}{|l|}{ Variety } \\
\hline Lydia & & & & $502+50 d$ & $8.05 \pm$ & & 51.4 & $71.0 \pm$ \\
\hline Carisma & $84.3 \pm 1.98 b$ & $4.27 \pm 0.27 \mathrm{c}$ & $12.80 \pm 0.83 \mathrm{~b}$ & $69.4 \pm 4.65^{\text {a }}$ & $7.84 \pm 0.65$ e & $277 \pm 22.08^{b}$ & $42.8 \pm 1.93 \mathrm{~d}$ & $74.2 \pm 3.45^{\mathrm{c}}$ \\
\hline Flash & $91.0 \pm 1.33^{\mathrm{a}}$ & $3.99 \pm 0.26^{\mathrm{d}}$ & $14.16 \pm 0.81^{a}$ & $71.3 \pm 4.11^{\mathrm{a}}$ & $8.52 \pm 0.58 b c$ & $292 \pm 22.08^{a}$ & $40.8 \pm 1.89 \mathrm{e}$ & $76.5 \pm 3.44^{\mathrm{a}}$ \\
\hline BA151 & $80.1 \pm 1.56^{\mathrm{c}}$ & $4.34 \pm 0.29^{c}$ & $11.92 \pm 0.78^{c}$ & $65.5 \pm 4.43 b c$ & $8.25 \pm 0.82^{\mathrm{cd}}$ & $292 \pm 23.09 \mathrm{a}$ & $47.0 \pm 2.12^{b}$ & $73.6 \pm 4.17^{\mathrm{c}}$ \\
\hline BA525 & $78.4 \pm 2.40^{\mathrm{d}}$ & $4.75 \pm 0.31 \mathrm{~b}$ & $11.02 \pm 0.77 \mathrm{~d}$ & $67.2 \pm 5.05 \mathrm{~b}$ & $8.87 \pm 0.85 \mathrm{~b}$ & $284 \pm 23.67 \mathrm{ab}$ & $45.2 \pm 2.05^{\mathrm{c}}$ & $73.7 \pm 3.46^{\mathrm{c}}$ \\
\hline ST468 & $84.9 \pm 1.35^{b}$ & $4.35 \pm 0.32^{c}$ & $13.03 \pm 0.86^{b}$ & $64.4 \pm 4.50^{\mathrm{c}}$ & $10.55 \pm 0.78^{a}$ & $291 \pm 21.05^{\mathrm{a}}$ & $42.1 \pm 1.88$ de & $75.6 \pm 3.51^{\mathrm{b}}$ \\
\hline
\end{tabular}

*: Means followed by same letter(s) in each column are not significant at $\mathrm{p}<0.05$. GP: Germination percentage, MGT: mean germination time, GI: Germination index, GSTI: Germination stress tolerance index, SL: Seedling length, SFW: Seedling fresh weight, SDW: Seedling dry weight, DM: Dry matter, SEM: Standart error of mean

The interaction of salinity $\times$ variety was significant for all characteristics $(\mathrm{p}<0.05)$ and the results of germination percentage were shown in Table 2. Germination percentage of cotton varieties was different in each salinity level under optimum and low temperature. It was declined with increasing $\mathrm{NaCl}$ and decreasing temperature. $\mathrm{NaCl}$ level of 100 $\mathrm{mM}$ inhibited germination of cotton varieties except for Flash and ST468. In addition, Flash and ST468 were the least affected varieties by salinity at $25^{\circ} \mathrm{C}$ and $18^{\circ} \mathrm{C}$. Adverse effects of salinity on germination were also observed by Ashraf et al. (2002), who observed the hazardous effects of $100 \mathrm{mM} \mathrm{NaCl}$ on germination percentage. Results of the current research showed that germination rate was considerably inhibited by low temperature. This finding was supported by Krzyzanowski and Delouche (2011) indicated that germination percentage dropped at $18^{\circ} \mathrm{C}$ and a significant reduction in germination rate from $\% 92.5$ at $24^{\circ} \mathrm{C}$ to $\% 81.2$ at $16^{\circ} \mathrm{C}$.

The most rapid mean time to germination was obtained from Flash at all $\mathrm{NaCl}$ levels except for 100 $\mathrm{mM}$ under optimum condition. Furthermore, it gave the minimum time to germination at $18^{\circ} \mathrm{C}$. Increasing salinity concentrations resulted in delaying germination and cvs. Carisma and BA151 showed faster mean time to germination after Flash compared to other varieties. Similar findings are in line with the results of Day et al. (2008) in sunflower, Kan et al. (2015) in soybean and Sarkar et al. (2019) in rice, who observed that mean germination time was delayed by increasing salinity.
Seedling length was severely depressed with increasing $\mathrm{NaCl}$ levels and decreasing temperature. At $25^{\circ} \mathrm{C}$, seedling length of Carisma and BA151 was not significantly affected up to $100 \mathrm{mM} \mathrm{NaCl}$. Similar results related with seedling length had been reported by Bauer and Bradow (1996), Javid et al. (2001), Ashraf et al. (2002) and Barpete et al. (2015) who found an apparent reduction in seedling growth of cotton due to salinity. However, the most sensitive variety to low temperature was Lydia because a dramatic reduction occurred between $18^{\circ} \mathrm{C}$ and $25^{\circ} \mathrm{C}$, Flash and ST468 produced longer seedling under low temperature. No seedling growth to be measured was observed at $250 \mathrm{mM}$ at $18^{\circ} \mathrm{C}$.

Seedling fresh weight of cotton varieties was obviously reduced as salinity increased and temperature decreased. Salinity level of $150 \mathrm{mM}$ led to depressing fresh weight of cotton seedling, while ST468 produced heavier seedling at $18^{\circ} \mathrm{C}$ and $25^{\circ} \mathrm{C}$ under $\mathrm{NaCl}$. Fresh weight of BA525 and ST468 at $25^{\circ} \mathrm{C}$ increased with $50 \mathrm{mM} \mathrm{NaCl}$ compared to control. Barpete et al. (2015) supported this finding by demonstrating a dramatic reduction in fresh weight of cotton seedlings under low temperature. Also, an apparent difference was observed the seedling growth between $18^{\circ} \mathrm{C}$ and $25^{\circ} \mathrm{C}$. This result agrees with Khan et al. (1995), Ashraf (2002) and Hanif et al. (2008). They reported that seedling fresh weight decreased in higher salt concentrations compared to control.

Increasing salinity caused considerably decreases in seedling dry weight (Table 3). Cotton varieties 
exhibited different responses to each salinity level and Lydia produced the highest dry weights in 200 $\mathrm{mM} \mathrm{NaCl}$ at $18^{\circ} \mathrm{C}$ and $250 \mathrm{mM}$ at $25^{\circ} \mathrm{C}$. At suboptimal temperature, Flash and Carisma gave the minimum dry weight at $200 \mathrm{mM} \mathrm{NaCl}$.

In previous researches, reduction of seedling fresh and dry weight under saline conditions in cotton had been reported by Javid et al. (2001), Hanif et al.
(2008) and Shaheen et al. (2012). Furthermore, low dose of $\mathrm{NaCl}$ resulted in a decline of dry matter in cotton seedlings. Higher dry matter production was achieved at $25^{\circ} \mathrm{C}$ than at $18^{\circ} \mathrm{C}$, but increasing salinity reduced dry matter of the varieties. Flash showed the superiority under low temperature, while ST468 and Flash accumulated heavier dry matter than the others at $25^{\circ} \mathrm{C}$.

Table 2. Germination percentage, mean germination time and seedling length of cotton varieties under low temperature and salinity stresses

Tablo 2. Düşük sıcaklık ve tuzluluk stresleri altında pamuk çeşitlerinin çimlenme yüzdesi, ortalama çimlenme süresi ve fide uzunluğu

\begin{tabular}{|c|c|c|c|c|c|c|c|c|}
\hline & \multirow{2}{*}{$\begin{array}{l}\text { Salinity } \\
(\mathrm{mM})\end{array}$} & \multicolumn{6}{|l|}{ Variety } \\
\hline & & & Lydia & Carisma & Flash & BA151 & BA525 & ST468 \\
\hline \multicolumn{9}{|c|}{ Germination percentage $(\%)_{ \pm}$SEM } \\
\hline \multirow{12}{*}{ 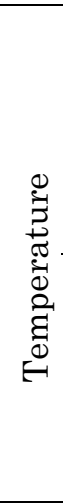 } & \multirow{6}{*}{$18^{\circ} \mathrm{C}$} & Cont. & $91.0 \pm 1.299^{\text {a-e }}$ & $93.0 \pm 1.29^{\mathrm{a}-\mathrm{d}}$ & $95.0 \pm 1.00 \mathrm{ab}$ & $92.0 \pm 0.82^{\text {a }}$ & $87.5 \pm 1.26^{b-f}$ & $91.5 \pm 0.50^{a-\mathrm{e}^{*}}$ \\
\hline & & 50 & $89.5 \pm 2.06^{\text {a }-\mathrm{e}}$ & $94.5 \pm 1.50 \mathrm{abc}$ & $97.0 \pm 1.29^{\mathrm{a}}$ & $86.5 \pm 1.71^{\mathrm{c} \cdot \mathrm{g}}$ & $84.5 \pm 1.71$ efg & $92.0 \pm 3.37^{\text {a e }}$ \\
\hline & & 100 & $80.0 \pm 2.45^{\mathrm{f}-\mathrm{j}}$ & $89.0 \pm 2.65^{\text {a-e }}$ & $96.0 \pm 1.41^{\mathrm{a}}$ & $79.0 \pm 2.65^{\mathrm{g} \cdot \mathrm{j}}$ & $73.0 \pm 3.46^{\mathrm{h}-\mathrm{k}}$ & $85.5 \pm 1.71^{\mathrm{d} \cdot \mathrm{g}}$ \\
\hline & & 150 & $80.0 \pm 3.46^{\mathrm{f} j \mathrm{j}}$ & $79.0 \pm 2.08 \mathrm{~g} \cdot \mathrm{j}$ & $94.0 \pm 1.63$ abc & $78.5 \pm 2.22_{\mathrm{g}-\mathrm{j}}$ & $69.0 \pm 1.29 \mathrm{k}$ & $81.0 \pm 3.00^{\mathrm{fgh}}$ \\
\hline & & 200 & $53.5 \pm 2.22^{\mathrm{m}}$ & $72.5 \pm 0.96^{\mathrm{jjk}}$ & $84.5 \pm 2.87 \mathrm{efg}$ & $72.0 \pm 2.16^{\mathrm{jk}}$ & $73.5 \pm 3.50^{\mathrm{h}-\mathrm{k}}$ & $80.5 \pm 3.30^{f-1}$ \\
\hline & & 250 & $14.5 \pm 1.26^{\circ}$ & $47.5 \pm 3.78 \mathrm{mn}$ & $67.5 \pm 4.50^{\mathrm{k}}$ & $52.5 \pm 3.30 \mathrm{~m}$ & $42.0 \pm 4.69^{\mathrm{n}}$ & $60.5 \pm 3.78^{1}$ \\
\hline & \multirow{6}{*}{$25^{\circ} \mathrm{C}$} & Cont. & $91.5 \pm 1.50^{\text {a-e }}$ & $93.0 \pm 2.38^{\mathrm{a}-\mathrm{d}}$ & $94.5 \pm 2.99 \mathrm{abc}$ & $88.5 \pm 0.96^{\mathrm{b}-\mathrm{g}}$ & $84.5 \pm 4.27^{\mathrm{d} \cdot 1}$ & $94.0 \pm 1.63$ abc \\
\hline & & 50 & $79.5 \pm 2.06$ ghr & $89.0 \pm 1.00^{\mathrm{a}-\mathrm{f}}$ & $96.5 \pm 0.96 \mathrm{ab}$ & $87.5 \pm 0.96^{\mathrm{b}-\mathrm{h}}$ & $87.5 \pm 2.22^{\mathrm{b}-\mathrm{h}}$ & $86.5 \pm 1.50^{c-1}$ \\
\hline & & 100 & $82.5 \pm 2.63^{\mathrm{e}-1}$ & $87.5 \pm 3.78^{\mathrm{b}-\mathrm{h}}$ & $96.0 \pm 1.63 \mathrm{ab}$ & $82.5 \pm 2.63^{\mathrm{c}^{-1}}$ & $86.5 \pm 2.87^{\mathrm{c}^{-1}}$ & $91.0 \pm 0.58^{\text {aee }}$ \\
\hline & & 150 & $83.5 \pm 6.65^{\mathrm{e}^{-1}}$ & $94.0 \pm 1.83$ abc & $90.0 \pm 1.41^{\text {a-e }}$ & $84.5 \pm 2.99^{\mathrm{d}-1}$ & $83.0 \pm 1.92^{e^{-1}}$ & $86.0 \pm 2.16^{\mathrm{c}^{-1}}$ \\
\hline & & 200 & $80.5 \pm 2.36^{f^{-1}}$ & $86.0 \pm 2.16^{\mathrm{c}-1}$ & $97.5 \pm 0.50^{a}$ & $79.0 \pm 4.80^{h-1}$ & $88.0 \pm 2.16^{\mathrm{b}-\mathrm{h}}$ & $87.5 \pm 0.50^{\mathrm{b}-\mathrm{h}}$ \\
\hline & & 250 & $79.0 \pm 2.08 \mathrm{hr}$ & $86.0 \pm 3.46^{\mathrm{c}^{-1}}$ & $83.5 \pm 2.57 \mathrm{e}^{-1}$ & $79.0 \pm 3.00^{\mathrm{hr}}$ & $77.5 \pm 4.72^{1}$ & $82.5 \pm 1.71^{\mathrm{e}^{-1}}$ \\
\hline \multicolumn{9}{|c|}{ Mean germination time (day) $\pm \mathrm{SEM}$} \\
\hline \multirow{12}{*}{ 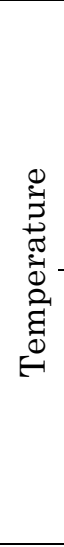 } & \multirow{6}{*}{$18^{\circ} \mathrm{C}$} & Cont. & $4.30 \pm 0.45^{\mathrm{hij}}$ & $3.59 \pm 0.29 \mathrm{kl}$ & $3.21 \pm 0.02^{1}$ & $3.73 \pm 0.30^{\mathrm{jkl}}$ & $4.34 \pm 0.34^{\mathrm{hij}}$ & $3.37 \pm 0.21^{\mathrm{kl}}$ \\
\hline & & 50 & $5.11 \pm 0.21^{\mathrm{fg}}$ & $3.87 \pm 0.05^{\mathrm{jk}}$ & $3.63 \pm 0.09 \mathrm{kl}$ & $3.72 \pm 0.03^{\mathrm{jkl}}$ & $4.26 \pm 0.25$ hij & $4.71 \pm 0.32^{\mathrm{gh}}$ \\
\hline & & 100 & $5.93 \pm 0.23 \mathrm{e}$ & $4.40 \pm 0.10^{\mathrm{hr}}$ & $4.29 \pm 0.12$ hij & $4.49 \pm 0.06^{\mathrm{hr}}$ & $4.64 \pm 0.10^{\mathrm{gh}}$ & $3.93 \pm 0.06^{\mathrm{ijk}}$ \\
\hline & & 150 & $7.29 \pm 0.43^{\mathrm{d}}$ & $5.35 \pm 0.17^{\mathrm{f}}$ & $5.24 \pm 0.09^{\mathrm{fg}}$ & $5.23 \pm 0.10^{\mathrm{fg}}$ & $6.25 \pm 0.05^{\mathrm{e}}$ & $5.23 \pm 0.07 \mathrm{fg}$ \\
\hline & & 200 & $8.70 \pm 0.18^{b}$ & $7.26 \pm 0.12^{\mathrm{d}}$ & $6.03 \pm 0.06 \mathrm{e}$ & $6.93 \pm 0.03^{\mathrm{d}}$ & $8.03 \pm 0.02^{c}$ & $7.37 \pm 0.10^{d}$ \\
\hline & & 250 & $9.63 \pm 0.18^{\mathrm{a}}$ & $8.49 \pm 0.37 \mathrm{bc}$ & $8.64 \pm 0.13^{b}$ & $9.34 \pm 0.21^{\mathrm{a}}$ & $9.42 \pm 0.15^{\mathrm{a}}$ & $9.82 \pm 0.16^{\text {a }}$ \\
\hline & \multirow{6}{*}{$25^{\circ} \mathrm{C}$} & Cont. & $3.15 \pm 0.21^{\mathrm{hr}}$ & $2.37 \pm 0.02^{\text {nop }}$ & $2.08 \pm 0.03^{p}$ & $2.19 \pm 0.02^{p}$ & $2.53 \pm 0.06^{1-0}$ & $2.16 \pm 0.06^{p}$ \\
\hline & & 50 & $3.43 \pm 0.13^{\mathrm{fgh}}$ & $2.42 \pm 0.08^{\mathrm{m}-\mathrm{p}}$ & $2.13 \pm 0.02^{\mathrm{p}}$ & $2.25 \pm 0.08^{\text {op }}$ & $2.52 \pm 010^{1-0}$ & $2.13 \pm 0.04^{\mathrm{p}}$ \\
\hline & & 100 & $3.26 \pm 0.12 \mathrm{ghr}$ & $2.37 \pm 0.05^{\text {nop }}$ & $2.94 \pm 0.02^{\mathrm{ijk}}$ & $2.94 \pm 0.04^{\mathrm{ijk}}$ & $2.72 \pm 0.05^{\mathrm{j}-\mathrm{m}}$ & $2.55 \pm 0.13^{\text {1.o }}$ \\
\hline & & 150 & $3.97 \pm 0.13^{\mathrm{e}}$ & $2.96 \pm 0.08^{\mathrm{ijk}}$ & $2.52 \pm 0.07^{1-0}$ & $2.82 \pm 0.04^{\mathrm{jjk}}$ & $2.97 \pm 0.09^{\mathrm{ij}}$ & $2.64 \pm 0.05^{\mathrm{k}-\mathrm{n}}$ \\
\hline & & 200 & $4.33 \pm 0.05^{\mathrm{d}}$ & $3.61 \pm 0.13^{\mathrm{f}}$ & $3.52 \pm 0.13^{\mathrm{fg}}$ & $3.93 \pm 0.11^{\mathrm{e}}$ & $3.94 \pm 0.11^{\mathrm{e}}$ & $3.61 \pm 0.07^{\mathrm{f}}$ \\
\hline & & 250 & $4.90 \pm 0.07^{b}$ & $4.63 \pm 0.35 \mathrm{bcd}$ & $3.67 \pm 0.09$ ef & $4.46 \pm 0.22^{\mathrm{cd}}$ & $5.43 \pm 0.08^{\mathrm{a}}$ & $4.73 \pm 0.24$ bc \\
\hline \multicolumn{9}{|c|}{ Seedling length $(\mathrm{cm}) \pm$ SEM } \\
\hline \multirow{12}{*}{ 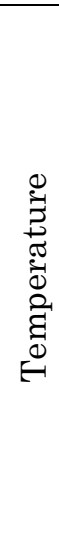 } & \multirow{6}{*}{$18^{\circ} \mathrm{C}$} & Cont. & $3.93 \pm 0.15^{\mathrm{jk}}$ & $7.14 \pm 0.24$ efg & $12.04 \pm 0.79^{b}$ & $6.21 \pm 0.33^{\mathrm{gh}}$ & $9.72 \pm 0.11^{d}$ & $14.88 \pm 0.58^{\mathrm{a}}$ \\
\hline & & 50 & $6.03 \pm 0.47^{\mathrm{gh}}$ & $6.33 \pm 0.45^{\mathrm{gh}}$ & $9.91 \pm 0.48^{\mathrm{d}}$ & $7.84 \pm 0.21^{\mathrm{e}}$ & $7.60 \pm 0.49^{\text {ef }}$ & $10.86 \pm 0.82^{\mathrm{c}}$ \\
\hline & & 100 & $4.65 \pm 0.28 \mathrm{klm}$ & $6.10 \pm 0.24^{\mathrm{hij}}$ & $8.89 \pm 0.33^{\mathrm{e}}$ & $5.31 \pm 0.17_{\mathrm{jkl}}$ & $6.06 \pm 0.41^{\mathrm{hij}}$ & $9.87 \pm 0.03^{\mathrm{d}}$ \\
\hline & & 150 & $3.93 \pm 0.15 \mathrm{mno}$ & $4.41 \pm 0.10^{\operatorname{lmn}}$ & $5.45 \pm 0.06^{\text {1jk }}$ & $4.64 \pm 0.09^{\mathrm{klm}}$ & $4.79 \pm 0.16^{\mathrm{klm}}$ & $6.66 \pm 0.36_{\mathrm{fgh}}^{\mathrm{fgh}}$ \\
\hline & & 200 & $2.79 \pm 0.05^{p}$ & $3.44 \pm 0.21$ nop & $3.94 \pm 0.13$ mno & $3.04 \pm 0.08_{\text {op }}$ & $2.97 \pm 0.10_{\text {op }}$ & $3.49 \pm 0.13^{\text {nop }}$ \\
\hline & & 250 & $-q$ & $-q$ & $-q$ & $-q$ & $-q$ & $-q$ \\
\hline & \multirow{6}{*}{$25^{\circ} \mathrm{C}$} & Cont. & $21.20 \pm 0.72^{b}$ & $12.62 \pm 0.29 \mathrm{hij}$ & $15.05 \pm 0.55^{\text {ef }}$ & $14.54 \pm 0.58^{\text {efg }}$ & $15.09 \pm 0.43^{\text {ef }}$ & $17.02 \pm 0.34^{\text {cd }}$ \\
\hline & & 50 & $16.48 \pm 0.63 \mathrm{de}$ & $13.43 \pm 0.52^{f-1}$ & $11.94 \pm 0.46^{\mathrm{ijk}}$ & $21.23 \pm 0.86^{\mathrm{b}}$ & $23.39 \pm 1.18^{\mathrm{a}}$ & $18.65 \pm 0.81^{\mathrm{c}}$ \\
\hline & & 100 & $12.98 \pm 0.40^{\text {gh }}$ & $16.37 \pm 1.42 \mathrm{de}$ & $9.79 \pm 0.35^{\mathrm{lm}}$ & $14.13 \pm 0.37^{\mathrm{fgh}}$ & $10.86 \pm 0.26^{\mathrm{jkl}}$ & $14.80 \pm 0.60^{\text {efg }}$ \\
\hline & & 150 & $9.13 \pm 0.64^{\mathrm{lmn}}$ & $10.54 \pm 0.51^{\mathrm{kl}}$ & $10.76 \pm 0.85^{\mathrm{jkl}}$ & $10.03 \pm 0.82^{\mathrm{klm}}$ & $10.86 \pm 0.26^{j \mathrm{kl}}$ & $13.54 \pm 0.88^{\mathrm{f}-1}$ \\
\hline & & 200 & $8.49 \pm 0.44 \mathrm{mno}$ & $7.59 \pm 0.25^{\text {nop }}$ & $7.82 \pm 0.39$ nор & $5.54 \pm 0.40^{q}$ & $9.88 \pm 0.17 \mathrm{~lm}$ & $9.91 \pm 0.67 \mathrm{~lm}$ \\
\hline & & 250 & $6.96 \pm 0.33$ opq & $6.10 \pm 0.39 \mathrm{pq}$ & $6.69 \pm 0.27$ орq & $6.34 \pm 0.51 \mathrm{pq}$ & $5.24 \pm 0.16^{q}$ & $6.98 \pm 0.70^{\mathrm{opq}}$ \\
\hline
\end{tabular}

*: Means followed by same letter(s) in each temperature are not significant at $\mathrm{p}<0.05$. Bracket (-) shows no data due to insufficient seedling growth. SEM: Standard error of mean. 
Table 3. Seedling fresh and dry weight and dry matter ratio of cotton varieties under low temperature and salinity stresses

Tablo 3. Düşük sıcaklık ve tuzluluk stresleri altında pamuk çeşitlerinin fide yaş ve kuru ă̆ırlı̆̆g ile kuru madde oranı

\begin{tabular}{|c|c|c|c|c|c|c|c|c|}
\hline & \multirow{2}{*}{$\begin{array}{l}\text { Salinity } \\
(\mathrm{mM})\end{array}$} & \multicolumn{6}{|l|}{ Variety } \\
\hline & & & Lydia & Carisma & Flash & BA151 & BA525 & ST468 \\
\hline \multicolumn{9}{|c|}{ Seedling fresh weight $\left(\mathrm{mg}\right.$ plant $\left.^{-1}\right) \pm \mathrm{SEM}$} \\
\hline \multirow{12}{*}{ 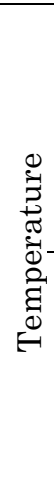 } & & Cont. & $314 \pm 15.31^{\mathrm{c}}$ & $291 \pm 6.87 \mathrm{~cd}$ & $362 \pm 15.05^{\text {a }}$ & $316 \pm 11.09^{c}$ & $297 \pm 6.56^{\text {cd }}$ & $358 \pm 7.86^{\mathrm{a}^{*}}$ \\
\hline & & 50 & $268 \pm 11.46$ de & $317 \pm 28.18^{\mathrm{c}}$ & $308 \pm 10.39^{c}$ & $352 \pm 12.07 \mathrm{ab}$ & $313 \pm 22.51^{\mathrm{c}}$ & $324 \pm 13.47$ bc \\
\hline & & 100 & $203 \pm 14.66^{\mathrm{h}}$ & $210 \pm 10.61 \mathrm{gh}$ & $240 \pm 4.12$ ef & $194 \pm 7.66^{\text {hı }}$ & $236 \pm 7.57 \mathrm{fg}$ & $250 \pm 4.37$ ef \\
\hline & & 150 & $157 \pm 2.92^{\mathrm{jk}}$ & $159 \pm 2.99 \mathrm{jk}$ & $157 \pm 6.03^{\mathrm{jkl}}$ & $172 \pm 4.41^{\mathrm{ij}}$ & $144 \pm 3.24^{\mathrm{j}-\mathrm{m}}$ & $162 \pm 8.40^{j}$ \\
\hline & & 200 & $146 \pm 3.80^{\mathrm{j}-\mathrm{m}}$ & $108 \pm 7.46^{\mathrm{n}}$ & $125 \pm 21.93 \mathrm{lmn}$ & $127 \pm 4.49^{\mathrm{k}-\mathrm{n}}$ & $123 \pm 5.42 \mathrm{mn}$ & $125 \pm 4.28^{\mathrm{lmn}}$ \\
\hline & & 250 & -0 & -0 & -0 & -0 & -0 & -0 \\
\hline & \multirow{6}{*}{$25^{\circ} \mathrm{C}$} & Cont. & $623 \pm 9.11^{\mathrm{a}}$ & $525 \pm 12.10$ def & $563 \pm 17.50^{\text {bcd }}$ & $575 \pm 11.72$ bc & $545 \pm 21.04$ cde & $497 \pm 14.83^{\mathrm{fg}}$ \\
\hline & & 50 & $448 \pm 11.47 \mathrm{hr}$ & $476 \pm 9.35^{\mathrm{gh}}$ & $500 \pm 11.68$ fg & $504 \pm 32.61 \mathrm{efg}$ & $604 \pm 7.79 \mathrm{ab}$ & $545 \pm 11.89$ cde \\
\hline & & 100 & $437 \pm 11.09 \mathrm{hl}$ & $473 \pm 22.48 \mathrm{gh}$ & $406 \pm 8.82^{1}$ & $458 \pm 19.80$ gh & $342 \pm 29.67^{j}$ & $405 \pm 14.01^{1}$ \\
\hline & & 150 & $244 \pm 19.02 \mathrm{mn}$ & $323 \pm 3.89 \mathrm{jk}$ & $333 \pm 20.33^{j k}$ & $328 \pm 16.68^{j k}$ & $317 \pm 6.69 \mathrm{jk}$ & $344 \pm 6.05^{j}$ \\
\hline & & 200 & $255 \pm 4.87 \mathrm{mn}$ & $263 \pm 8.79 \mathrm{lmn}$ & $287 \pm 8.58 \mathrm{klm}$ & $262 \pm 7.50^{\mathrm{lmn}}$ & $303 \pm 14.02^{\mathrm{jkl}}$ & $245 \pm 9.48 \mathrm{mn}$ \\
\hline & & 250 & $234 \pm 15.43^{\mathrm{n}}$ & $179 \pm 9.55^{\circ}$ & $227 \pm 3.44^{\mathrm{n}}$ & $217 \pm 9.68^{\text {nо }}$ & $180 \pm 7.66^{\circ}$ & $234 \pm 16.56^{\mathrm{n}}$ \\
\hline \multicolumn{9}{|c|}{ Seedling dry weight $\left(\mathrm{mg}^{2}\right.$ plant $\left.^{-1}\right) \pm \mathrm{SEM}$} \\
\hline \multirow{12}{*}{ 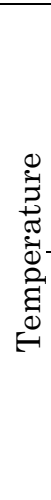 } & & Cont. & $59.4 \pm 1.85^{\mathrm{a}}$ & $49.9 \pm 1.16^{\mathrm{c}-\mathrm{g}}$ & $45.4 \pm 1.97$ ghr & $52.2 \pm 1.25^{\mathrm{cd}}$ & $52.1 \pm 1.03$ cde & $41.6 \pm 1.46^{1 \mathrm{jk}}$ \\
\hline & & 50 & $57.8 \pm 0.76$ ab & $49.4 \pm 1.85^{\mathrm{d}-\mathrm{g}}$ & $45.2 \pm 0.97$ ghı & $52.3 \pm 1.61 \mathrm{~cd}$ & $50.7 \pm 1.68^{\mathrm{c}-\mathrm{f}}$ & $48.0 \pm 0.92^{\mathrm{d}-g}$ \\
\hline & $18^{\circ} \mathrm{C}$ & 100 & $57.3 \pm 2.52^{\mathrm{ab}}$ & $46.8 \pm 1.75^{\mathrm{fgh}}$ & $48.2 \pm 1.27 \mathrm{~d} \cdot \mathrm{g}$ & $47.4 \pm 1.29$ efg & $49.7 \pm 1.53^{\mathrm{c}-\mathrm{g}}$ & $47.4 \pm 1.22 \mathrm{efg}$ \\
\hline & & 150 & $59.3 \pm 0.44^{\mathrm{a}}$ & $49.0 \pm 1.51^{\mathrm{d}-\mathrm{g}}$ & $40.5 \pm 1.74^{\mathrm{jk}}$ & $54.1 \pm 2.28$ bc & $42.6 \pm 0.87$ hij & $47.9 \pm 0.87^{\mathrm{d}-\mathrm{g}}$ \\
\hline & & 200 & $56.9 \pm 1.54^{\mathrm{ab}}$ & $39.1 \pm 0.51^{\mathrm{k}}$ & $33.3 \pm 0.88^{1}$ & $50.2 \pm 1.82^{\mathrm{c}-\mathrm{f}}$ & $51.9 \pm 2.83$ cde & $48.1 \pm 1.31^{\mathrm{d}-\mathrm{g}}$ \\
\hline & & 250 & $-\mathrm{m}$ & $-\mathrm{m}$ & $-\mathrm{m}$ & $-\mathrm{m}$ & $-\mathrm{m}$ & $-\mathrm{m}$ \\
\hline & \multirow{6}{*}{$25^{\circ} \mathrm{C}$} & Cont. & $53.8 \pm 2.03^{\mathrm{a}-\mathrm{d}}$ & $45.3 \pm 1.21$ ghı & $43.5 \pm 0.71^{1}$ & $48.9 \pm 1.02^{c^{-1}}$ & $49.1 \pm 1.22^{\mathrm{c}-1}$ & $45.0 \pm 2.26 \mathrm{gh}$ \\
\hline & & 50 & $56.3 \pm 2.75^{\mathrm{ab}}$ & $45.3 \pm 1.44^{\mathrm{gh}}$ & $47.0 \pm 1.20^{\mathrm{f}-1}$ & $49.5 \pm 0.50^{c^{-1}}$ & $50.5 \pm 1.20^{\mathrm{a}-\mathrm{h}}$ & $44.3 \pm 1.92 \mathrm{hr}$ \\
\hline & & 100 & $54.7 \pm 2.24^{\mathrm{abc}}$ & $46.3 \pm 0.72^{\text {ghl }}$ & $45.5 \pm 0.77 \mathrm{gh}$ & $53.0 \pm 0.97^{\mathrm{a}-\mathrm{f}}$ & $48.6 \pm 2.15^{\mathrm{c}-1}$ & $44.6 \pm 1.42^{\mathrm{hl}}$ \\
\hline & & 150 & $51.7 \pm 3.99^{\mathrm{a}-\mathrm{g}}$ & $48.1 \pm 0.74^{\mathrm{d}-1}$ & $47.9 \pm 1.30^{\mathrm{d}-1}$ & $53.3 \pm 1.01^{\mathrm{a}-\mathrm{f}}$ & $45.5 \pm 3.40 \mathrm{gh}$ & $45.8 \pm 1.11^{\mathrm{gh}}$ \\
\hline & & 200 & $56.6 \pm 2.78^{\mathrm{a}}$ & $49.3 \pm 0.40^{\mathrm{c}-1}$ & $47.0 \pm 1.50^{f-1}$ & $53.7 \pm 1.16^{\mathrm{a}-\mathrm{e}}$ & $51.8 \pm 0.76^{\mathrm{a-g}}$ & $47.1 \pm 3.17$ e-1 $^{-1}$ \\
\hline & & 250 & $53.1 \pm 2.19 \mathrm{a}-\mathrm{f}$ & $46.2 \pm 2.02 \mathrm{gh}$ & $45.9 \pm 1.36$ ghı & $48.8 \pm 5.57^{c^{-1}}$ & $49.8 \pm 1.67^{b-1}$ & $45.4 \pm 1.66 \mathrm{ghl}$ \\
\hline \multicolumn{9}{|c|}{ Dry matter $(\%) \pm$ SEM } \\
\hline \multirow{12}{*}{\multicolumn{2}{|c|}{ 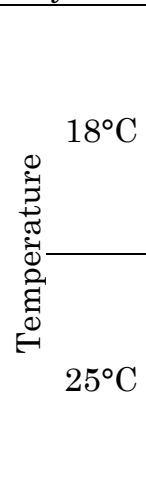 }} & Cont. & $79.2 \pm 2.86^{\text {fgh }}$ & $82.9 \pm 0.41$ cde & $87.2 \pm 1.07 \mathrm{ab}$ & $83.4 \pm 0.53 \mathrm{~cd}$ & $82.5 \pm 0.52 \mathrm{c}-\mathrm{f}$ & $87.6 \pm 1.21^{\mathrm{a}}$ \\
\hline & & 50 & $78.3 \pm 0.76$ ghı & $84.1 \pm 1.34$ bcd & $85.3 \pm 0.53 \mathrm{abc}$ & $85.0 \pm 0.65^{a b c}$ & $83.5 \pm 1.51^{\mathrm{cd}}$ & $85.1 \pm 0.83 \mathrm{abc}$ \\
\hline & & 100 & $71.8 \pm 0.55 \mathrm{klm}$ & $77.6 \pm 0.79 \mathrm{hı}$ & $80.0 \pm 0.25^{\mathrm{e}-\mathrm{h}}$ & $75.5 \pm 0.46^{\mathrm{ij}}$ & $79.1 \pm 0.75^{\text {fgh }}$ & $81.1 \pm 0.33^{\mathrm{d}-\mathrm{g}}$ \\
\hline & & 150 & $62.3 \pm 0.67$ no & $69.1 \pm 1.10^{\mathrm{lm}}$ & $74.2 \pm 0.51^{\mathrm{jk}}$ & $68.5 \pm 0.55^{\mathrm{m}}$ & $70.5 \pm 1.10^{\mathrm{lm}}$ & $72.0 \pm 0.67 \mathrm{kl}$ \\
\hline & & 200 & $61.0 \pm 1.28$ op & $64.3 \pm 2.31^{\mathrm{n}}$ & $71.5 \pm 3.48 \mathrm{klm}$ & $60.4 \pm 0.09$ op & $57.9 \pm 0.59^{\circ}$ & $61.6 \pm 0.64$ no \\
\hline & & 250 & $-q$ & $-\mathrm{q}$ & $-q$ & $-q$ & $-q$ & $-q$ \\
\hline & & Cont. & $91.4 \pm 0.27 \mathrm{ab}$ & $91.4 \pm 0.23 \mathrm{ab}$ & $92.3 \pm 0.16^{\text {a }}$ & $91.5 \pm 0.20^{\mathrm{ab}}$ & $91.0 \pm 0.22 \mathrm{abc}$ & $90.9 \pm 0.60 \mathrm{abc}$ \\
\hline & & 50 & $87.5 \pm 0.46^{\mathrm{d}-\mathrm{g}}$ & $90.5 \pm 0.37 \mathrm{abc}$ & $90.6 \pm 0.42^{a b c}$ & $89.6 \pm 0.50^{a-d}$ & $91.4 \pm 0.20 \mathrm{ab}$ & $92.0 \pm 0.35^{\mathrm{a}}$ \\
\hline & & 100 & $87.4 \pm 0.40^{\mathrm{d}-\mathrm{g}}$ & $90.2 \pm 0.38^{\mathrm{a}-\mathrm{d}}$ & $88.8 \pm 0.42^{b-e}$ & $88.4 \pm 0.61^{\mathrm{c}-\mathrm{f}}$ & $86.6 \pm 1.69$ efg & $89.0 \pm 0.17$ b-e \\
\hline & & 150 & $78.8 \pm 0.54^{\mathrm{kl}}$ & $85.1 \pm 0.39 \mathrm{gh}$ & $85.5 \pm 0.85^{\text {fgh }}$ & $83.7 \pm 0.65^{\mathrm{hl}}$ & $85.7 \pm 0.92^{\mathrm{fgh}}$ & $86.7 \pm 0.49 \mathrm{efg}$ \\
\hline & & 200 & $77.2 \pm 1.63^{1}$ & $81.2 \pm 0.69^{\mathrm{jjk}}$ & $83.6 \pm 0.44^{\mathrm{hl}}$ & $79.5 \pm 0.61^{\mathrm{kl}}$ & $82.8 \pm 0.67 \mathrm{hij}$ & $80.7 \pm 0.75^{\mathrm{jk}}$ \\
\hline & & 250 & $77.1 \pm 0.73^{1}$ & $74.0 \pm 1.24 \mathrm{~m}$ & $79.8 \pm 0.70 \mathrm{kl}$ & $77.3 \pm 3.07^{1}$ & $73.7 \pm 1.30 \mathrm{~m}$ & $80.4 \pm 1.49^{\mathrm{jk}}$ \\
\hline
\end{tabular}

*: Means followed by same letter(s) in each temperature are not significant at $\mathrm{p}<0.05$. Bracket (-) shows no data due to insufficient seedling growth. SEM: Standard error of mean

Vigor index of cotton varieties was severely influenced by each salinity level under optimal and low temperatures. It was higher at $25^{\circ} \mathrm{C}$ compared to $18^{\circ} \mathrm{C}$, but increased $\mathrm{NaCl}$ reduced vigor index. Vigor index could not be calculated in $\mathrm{NaCl}$ level of $250 \mathrm{mM}$ because of no seedling development. The highest value was obtained in Flash and ST468 under all salinity levels at $18^{\circ} \mathrm{C}$ (Table 4 ). These results agree with Liu et al. (2010) in sunflower, Kandil et al. (2012) and El Naim et al. (2012) in sorghum, and
Çarpıcı et al. (2009) in maize, they indicated that salinity and low temperature severely influenced vigor index with significant reduction.

The germination index of cotton varieties declined as salt levels increased. However, cotton varieties showed different responses to $\mathrm{NaCl}$ both at $18^{\circ} \mathrm{C}$ and at $25^{\circ} \mathrm{C}$. The highest germination index was attained in Flash and ST468 (Fig. 1). Also, Wang et al. (2007) determined that no significant change in germination index was observed in low salinity but drastically 
decreased as salinity increased. Similarly, germination stress tolerance index reduced with increasing salinity and low temperature. Among cotton varieties, Flash was the least affected variety by salinity under low temperature (Fig. 2).

The germination stress tolerance index drastically decreased due to increasing $\mathrm{NaCl}$ doses and this finding was reported in several crops by Karimi et al. (2011) in safflower, Abbaszadeh et al. (2012) in rapeseed, Kausar et al. (2012) in sorghum, and Vibhuti et al. (2015) in rice.

Table 4. Vigor index of cotton varieties under low temperature and salinity stresses $( \pm \mathrm{SEM})$

Tablo 4. Düşük sıcaklık ve tuzluluk stresleri altında pamuk çeşitlerinin güç indeksi ( ${ }_{\text {SStandart hata) }}$

\begin{tabular}{|c|c|c|c|c|c|c|c|}
\hline & \multirow{2}{*}{$\begin{array}{l}\text { Salinity } \\
(\mathrm{mM})\end{array}$} & \multicolumn{6}{|l|}{ Variety } \\
\hline & & Lydia & Carisma & Flash & BA151 & BA525 & ST468 \\
\hline \multirow{12}{*}{ 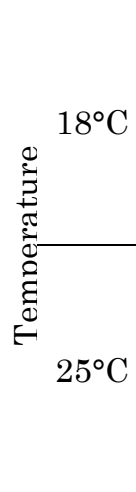 } & Cont. & $357 \pm 16.0^{\mathrm{jjk}}$ & $664 \pm 27.9$ e & $1142 \pm 71.3^{b}$ & $571 \pm 32.0^{\mathrm{fg}}$ & $850 \pm 11.5^{\mathrm{d}}$ & $1361 \pm 50.4 \mathrm{a}^{*}$ \\
\hline & 50 & $540 \pm 47.7 \mathrm{~g}$ & $600 \pm 50.0$ efg & $961 \pm 48.6^{c}$ & $677 \pm 21.6$ e & $643 \pm 50.0$ ef & $996 \pm 75.8^{c}$ \\
\hline & 100 & $372 \pm 25.3^{\mathrm{j} \mathrm{jk}}$ & $541 \pm 9.4 \mathrm{~g}$ & $853 \pm 24.5^{\mathrm{d}}$ & $421 \pm 26.1^{\mathrm{ij}}$ & $442 \pm 32.1 \mathrm{hr}$ & $844 \pm 17.6^{\mathrm{d}}$ \\
\hline & 150 & $315 \pm 22.5^{\mathrm{kl}}$ & $348 \pm 12.1^{\mathrm{ijk}}$ & $513 \pm 14.0 \mathrm{gh}$ & $363 \pm 9.6 \mathrm{jjk}$ & $331 \pm 14.1^{\mathrm{jkl}}$ & $541 \pm 44.2^{\mathrm{g}}$ \\
\hline & 200 & $149 \pm 5.0^{\mathrm{n}}$ & $248 \pm 13.3 \mathrm{~lm}$ & $331 \pm 4.0 \mathrm{jkl}$ & $219 \pm 9.8 \mathrm{mn}$ & $218 \pm 16.6 \mathrm{mn}$ & $305 \pm 24.3 \mathrm{klm}$ \\
\hline & 250 & -0 & -0 & -0 & -0 & -0 & -0 \\
\hline & Cont. & $1942 \pm 96.4 \mathrm{ab}$ & $1173 \pm 41.2 \mathrm{ght}$ & $1426 \pm 92.8$ def & $1302 \pm 62.4^{\mathrm{fg}}$ & $1279 \pm 92.5^{\mathrm{fg}}$ & $1600 \pm 39.4^{\text {cd }}$ \\
\hline & 50 & $1286 \pm 48.6^{\mathrm{fg}}$ & $1195 \pm 54.3 \mathrm{gh}$ & $1152 \pm 46.1^{\text {ghr }}$ & $1834 \pm 70.8^{b}$ & $2095 \pm 71.8^{a}$ & $1616 \pm 93.5^{\mathrm{c}}$ \\
\hline & 100 & $1071 \pm 50.1^{\text {hij }}$ & $1488 \pm 99.0$ cde & $941 \pm 49.2^{\mathrm{jkl}}$ & $1164 \pm 36.2$ ghr & $941 \pm 49.3^{\mathrm{jkl}}$ & $1346 \pm 48.8$ efg \\
\hline & 150 & $797 \pm 75.6^{\mathrm{k}-\mathrm{n}}$ & $992 \pm 62.5^{\mathrm{ijk}}$ & $965 \pm 65.4^{\mathrm{jk}}$ & $876 \pm 63.8^{j-m}$ & $902 \pm 41.2^{j \mathrm{kl}}$ & $1194 \pm 75.6 \mathrm{gh}$ \\
\hline & 200 & $686 \pm 53.0 \mathrm{~m}-\mathrm{p}$ & $653 \pm 32.5^{\text {nop }}$ & $762 \pm 34.1^{1-o}$ & $443 \pm 57.8$ q & $870 \pm 35.1 \mathrm{klm}$ & $842 \pm 37.0 \mathrm{k}-\mathrm{n}$ \\
\hline & 250 & $552 \pm 40.3 \mathrm{pq}$ & $528 \pm 52.9 \mathrm{pq}$ & $556 \pm 11.4 \mathrm{pq}$ & $505 \pm 58.5$ pq & $406 \pm 30.4 \mathrm{q}$ & $577 \pm 63.5$ opq \\
\hline
\end{tabular}

*: Means followed by same letter(s) in each temperature are not significant at $\mathrm{p}<0.05$. Bracket (-) shows no data due to insufficient seedling growth. SEM: Standard error of mean

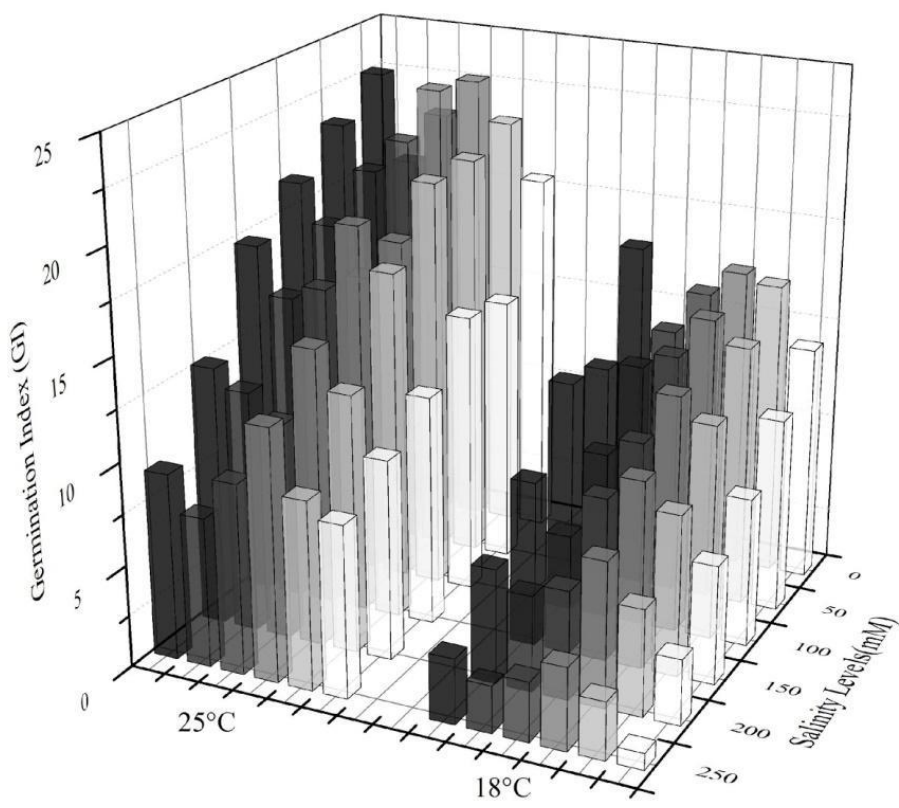

Figure 1. Germination index of cotton varieties under low temperature and salinity stresses Şekil 1. Düşük sıcaklık ve tuzluluk stresleri altında pamuk çeşitlerinin çimlenme indeksi 


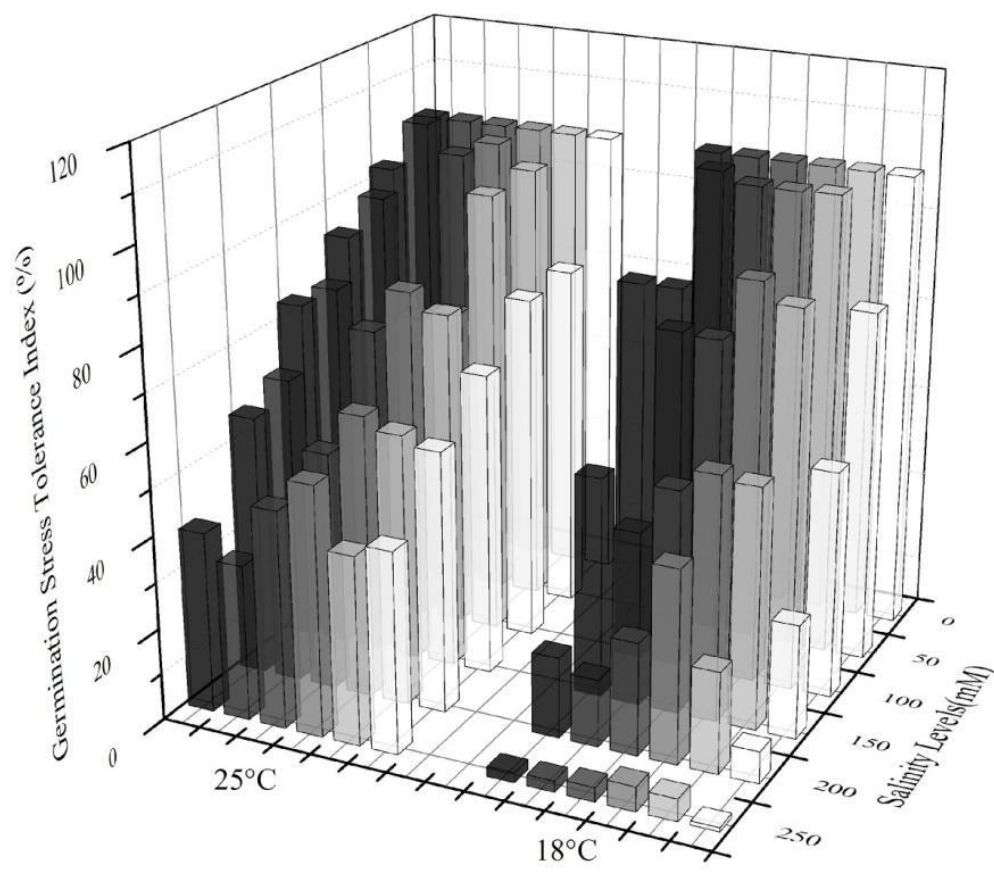

Figure 2. Germination stress tolerance index of cotton varieties under low temperature and salinity stresses Şekil 2. Düşük sıcaklık ve tuzluluk stresleri altında pamuk çeşitlerinin çimlenme stres tolerans indeksi

\section{Statement of Conflict of Interest}

Authors have declared no conflict of interest.

\section{Author's Contributions}

The contribution of the authors is equal.

\section{REFERENCES}

Abbaszadeh F, Rameeh V, Cherati A 2012. Salinity Stress Indices of Seed Yield and Nutrient Compositions in Rapeseed (Brassica napus L.). International Journal of Biology 4(1): 154. http://dx.doi.org/10.5539/ijb.v4n1p154

Anonymous, 2020. https://biruni.tuik.gov.tr/medas/ $? \mathrm{kn}=92 \&$ locale $=$ tr

Ahmad S, Ahmad R, Ashraf MY, Ashraf M, Waraich EA 2009. Sunflower (Helianthus annuus L.) Response to Drought Stress at Germination and Seedling Growth Stages. Pakistan Journal of Botany 41(2): 647-654.

Ashraf M 2002. Salt Tolerance of Cotton: Some New Advances. Critical Reviews in Plant Sciences 21(1): 1-30. https://doi.org/10.1080/0735-260291044160

Ashraf MY, Sarwar G, Ashraf M, Afaf R, Sattar A 2002. Salinity Induced Changes in a-amylase Activity During Germination and Early Cotton Seedling Growth. Biologia Plantarum 45(4): 589591. https://doi.org/10.1023/A:1022338900818

Barpete S, Oğuz MC, Özcan SF, Anayol E, Ahmed HA, Khawar KM, Özcan S 2015. Effect of Temperature on Germination, Seed Vigor Index and Seedling Growth of Five Turkish Cotton
(Gossypium hirsutum L.) Cultivars. Fresenius Environmental Bulletin 24: 2561-2566.

Bauer PJ, Bradow JM 1996. Cotton genotype response to early-season cold temperatures. Crop Science 36(6): 1602-1606. https://doi.org/10.2135/ cropsci1996.0011183X003600060032x

Çarpıcı EB, Çelik N, Bayram G 2009. Effects of Salt Stress on Germination of Some Maize (Zea mays L.) Cultivars. African Journal of Biotechnology, 8(19): 4918-4922.

Çokkizgin H, Bölek Y 2015. Priming Treatments for Improvement of Germination and Emergence of Cotton Seeds at Low Temperature. Plant Breeding and Seed Science 71(1): 121-134. https://doi.org/10.1515/plass-2015-0027

Day S, Kaya MD, Kolsaricı Ö 2008. Effects of $\mathrm{NaCl}$ Levels on Germination of Some Confectionary Sunflower (Helianthus annuus L.) Genotypes. Journal of Agricultural Sciences 4(3): 230-236. (in Turkish).

El Naim AM, Mohammed KE, Ibrahim EA, Suleiman NN 2012. Impact of Salinity on Seed Germination and Early Seedling Growth of Three Sorghum (Sorghum biolor L. Moench) Cultivars. Science and Technology 2(2): 16-20. http://doi.org/10.5923/ j.scit. 20120202.03

Fowler JL 1986. Salinity and fruiting. (Cotton physiology. The Cotton Foundation, Memphis, TN) 107-111.

Gossett DR., Millhollon EP, Lucas MC 1994. Antioxidant Response to $\mathrm{NaCl}$ Stress in Salt- 
Tolerant and Salt-Sensitive Cultivars of Cotton. Crop Science 34: 706-714. https://doi.org/10.2135/ cropsci1994.0011183X003400030020x

Hanif M, Noor E, Murtaza N, Qayyum A, Malik W 2008. Assessment of Variability for Salt Tolerance at Seedling Stage in Gossypium hirsutum L. Journal of Food Agriculture and Environment 6(1): 134.

ISTA 2003. International Rules For Seed Testing. International Seed Testing Association, Bassersdorf, Switzerland.

Jackson MB 2012. New root formation in plants and cuttings (Vol. 20). (Springer Science \& Business Media. Dordrecth, The Netherlands) http://dx.doi.org/10.1007/978-94-009-4358-2

Javid A, Yasin M, Nabi G 2001. Effect of Seed PreTreatments on Germination and Growth of Cotton (Gossypium hirsutum L) Under Saline Conditions. Pakistan Journal of Biological Sciences 4(9): 11081110

Kalefetoğlu T, Ekmekçi Y 2005. The Effects of Drought on Plants and Tolerance Mechanisms. Gazi University Journal of Science, 18(4): 723-740.

Kan G, Zhang W, Yang W, Ma D, Zhang D, Hao D, Hu Z, Yu D 2015. Association Mapping of Soybean Seed Germination Under Salt Stress. Molecular Genetics and Genomics 290(6): 2147-2162. https://doi.org/10.1007/s00438-015-1066-y

Kandil AA, Sharief AE, Abido WAE, Ibrahim MM 2012. Effect of Salinity on Seed Germination and Seedling Characters of Some Forage Sorghum Cultivars. International Journal of Agriculture Sciences 4(7): 306.

Karimi N, Soheilikhah Z, Ghasmpour HR, Zebarjadi A 2011. Effect of Salinity Stress on Germination and Early Seedling Growth of Different Safflower (Carthamus tinctorius L.) Genotypes. Journal of Ecobiotechnology 3(10): 07-13.

Kausar A, Ashraf MY, Ali I, Niaz M, Abbass Q 2012. Evaluation of Sorghum Varieties/Lines for Salt Tolerance Using Physiological Indices as Screening Tool. Pakistan Journal of Botany 44(1): 47-52.

Khajeh-Hosseini M, Powell AA, Bingham IJ 2003. The Interaction Between Salinity Stress and Seed Vigour During Germination of Soybean Seeds. Seed Science and Technology 31(3): 715-725. https://doi.org/10.15258/sst.2003.31.3.20

Khan AN, Qureshi RH, Ahmad N 1995. Selection of cotton cultivars for Salinity Tolerance at Seedling Stage. Sarhad Journal of Agriculture 11(2): 153159.
Krzyzanowski FC, Delouche JC 2011. Germination of Cotton Seed in Relation to Temperature. Revista Brasileira de Sementes 33(3): 543-548. https://doi.org/10.1590/S0101-31222011000300017

Liu J, Guo WQ, Shi DC 2010. Seed Germination, Seedling Survival, and Physiological Response of Sunflowers Under Saline and Alkaline Conditions. Photosynthetica 48(2): 278-286. https://doi.org/ 10.1007/ s11099-010-0034-3

Maas EV 1986. Salt Tolerance of Plants. Journal of Applied Agricultural Research 1(1): 12-25.

Mert M 2017. Fiber Crops. (Nobel Akademic Press. 2nd Ed) 448 p. (in Turkish).

Qadir M, Shams M 1997. Some Agronomic and physiological aspects of Salt Tolerance in Cotton (Gossypium hirsutum L.). Journal of Agronomy and Crop Science 179: 101-106. https://doi.org/10.1111/j.1439-037X.1997.tb00504.x

Salehzade H, Shishvan MI, Ghiyasi M, Forouzin F, Siyahjani AA 2009. Effect of Seed Priming on Germination and Seedling Growth of Wheat (Triticum aestivum L.). Reseach Journal of Biological Sciences 4(5): 629-631.

Sarkar MN, Hossain AZ, Begum S, Islam SN, Biswas SK, Tareq MZ 2019. Effect of Salinity on Seed Germination and Seedlings Growth of Sorghum (Sorghum bicolor L.). Journal of Bioscience and Agriculture Research 21(02): 1786-1793. https://doi.org/10.18801/jbar.210219.218

Shaheen HL, Shahbaz M, Ullah I, Iqbal MZ 2012. Morpho-physiological Responses of Cotton (Gossypium hirsutum) to Salt Stress. International Journal of Agriculture and Biology 14(6): 980-984.

Varghese S, Patil KV, Gohil MD, Bhatt PH, Patil UG 1995. Response of G-COT-11 Levant Cotton (Gossypium herbaceum) to Salinity at Germination Stage. Indian Journal of Agricultural Sciences 65: 823-825.

Vibhuti CS, Bargali K, Bargali SS 2015. Seed Germination and Seedling Growth Parameters of Rice (Oryza sativa L.) Varieties as Affected by Salt and Water Stress. Indian Journal of Agricultural Sciences 85(1): 102-108.

Vulkan-Levy R, Ravina I, Mantell A, Frenkel H 1998. Effect of Water Supply and Salinity on Pima Cotton. Agricultural Water Management 37(2): 121-132. https://doi.org/10.1016/S03783774(98)00042-0

Wang JJ, Ye WW, Zhou DY, Lü YJ, Fan BX, Song LY 2007. Studies on Germination Characteristics of Different Salinity-Resistant Cotton Under Salt Stress. Cotton Science 4. 